\title{
Autophagy Plays an Important Role in Anti-inflammatory Mechanisms Stimulated by Alpha7 Nicotinic Acetylcholine Receptor
}

\author{
Bo-Zong Shao ${ }^{1 t}$, Ping Ke ${ }^{1 t}$, Zhe-Qi Xu', Wei Wei ${ }^{2}$, Ming-He Cheng ${ }^{1}$, Bin-Ze Han', \\ Xiong-Wen Chen ${ }^{3}$, Ding-Feng Su ${ }^{1 *}$ and Chong Liu ${ }^{1 *}$
}

${ }^{1}$ Department of Pharmacology, Second Military Medical University, Shanghai, China, ${ }^{2}$ Institute of Quality and Standard for Agro-Products, Zhejiang Academy of Agricultural Sciences, Hangzhou, China, ${ }^{3}$ Cardiovascular Research Center, Temple University School of Medicine, Philadelphia, PA, USA

OPEN ACCESS

Edited by:

Kai Fang,

University of California at Los

Angeles, USA

Reviewed by:

Maryna Skok,

Palladin Institute of Biochemistry,

Ukraine

Agustina Alaimo,

University of Buenos Aires, Argentina

*Correspondence:

Chong Liu

wanlc2004@aliyun.com;

Ding-Feng Su

dfsu2008@gmail.com

these authors have contributed equally to this work.

Specialty section:

This article was submitted to

Inflammation,

a section of the journal

Frontiers in Immunology

Received: 13 March 2017

Accepted: 25 April 2017

Published: 16 May 2017

Citation:

Shao B-Z, Ke P, Xu Z-Q, Wei W,

Cheng $M-H$, Han B-Z, Chen X-W,

Su D-F and Liu C (2017) Autophagy

Plays an Important Role in

Anti-inflammatory Mechanisms

Stimulated by Alpha7 Nicotinic

Acetylcholine Receptor.

Front. Immunol. 8:553.

doi: 10.3389/fimmu.2017.00553
Alpha7 nicotinic acetylcholine receptor $(\alpha 7 \mathrm{nAChR})$ has been reported to alleviate neuroinflammation. Here, we aimed to determine the role of autophagy in $\alpha 7 n A C h R-$ mediated inhibition of neuroinflammation and its underlying mechanism. Experimental autoimmune encephalomyelitis (EAE) mice and lipopolysaccharide-stimulated BV2 microglia were used as in vivo and in vitro models of neuroinflammation, respectively. The severity of EAE was evaluated with neurological scoring. Autophagy-related proteins (Beclin 1, LC3-II/I, p62/SQSTM1) were detected by immunoblot. Autophagosomes were observed using transmission electron microscopy and tandem fluorescent mRFPGFP-LC3 plasmid was applied to test autophagy flux. The mRNA levels of interleukin-6 (IL-6), IL-1 $\beta, I L-18$, and tumor necrosis factor- $\alpha$ (TNF- $\alpha$ ) were detected by real-time PCR. We used 3-methyladenine (3-MA) and autophagy-related gene 5 small interfering RNA (Atg5 siRNA) to block autophagy in vivo and in vitro, respectively. Activating $\alpha 7 \mathrm{nAChR}$ with PNU282987 ameliorates EAE severity and spinal inflammatory infiltration in EAE mice. PNU282987 treatment also enhanced monocyte/microglia autophagy (Beclin 1, LC3-II/I ratio, p62/SQSTM1, colocalization of CD45- or CD68-positive cells with LC3) both in spinal cord and spleen from EAE mice. The beneficial effects of PNU282987 on EAE mice were partly abolished by 3-MA, an autophagy inhibitor. In vitro, PNU282987 treatment increased autophagy and promoted autophagy flux. Blockade of autophagy by Atg5 siRNA or bafilomycin A1 attenuated the inhibitory effect of PNU282987 on IL-6, $\mathrm{IL}-1 \beta, \mathrm{IL}-18$, and TNF- $\alpha \mathrm{mRNA}$. Our results demonstrate for the first time that activating $\alpha 7 n A C h R$ enhances monocyte/microglia autophagy, which suppresses neuroinflammation and thus plays an alleviative role in EAE.

Keywords: alpha7 nicotinic acetylcholine receptor, autophagy, neuroinflammation, experimental autoimmune encephalomyelitis, microglia

\section{INTRODUCTION}

It is commonly believed that microglia contribute to the triggering of inflammatory responses in central nervous system (CNS), mainly through the induction of inflammatory cytokine production as well as the immune responses $(1,2)$. However, the overactivation of microglia may lead to the pathogenesis and aggravation of CNS damage in inflammatory diseases including multiple 
sclerosis (MS) (3-5). MS is recognized as a chronic inflammatory autoimmune disorder featuring CNS demyelination and neurodegeneration $(6,7)$. Accumulation and overactivation of microglia greatly aggravates the severity of symptoms and demyelination in experimental autoimmune encephalomyelitis (EAE) mice $(8,9)$. Thus, suppressing microglia-mediated inflammation serves as a potential therapeutic strategy for MS or EAE.

Alpha7 nicotinic acetylcholine receptor $(\alpha 7 \mathrm{nAChR})$ is a subtype of nAChRs, which is a member of superfamily of cysloop cationic ligand-gated channels $(10,11)$. Studies from our lab previously showed that $\alpha 7 \mathrm{nAChR}$ was associated with various cardiovascular diseases $(12,13)$. Recently, there is evidence that activation of $\alpha 7 \mathrm{nAChR}$ contributes to the alleviation of neuroinflammation in EAE model (14-16), but the underlying mechanism has not been fully clarified.

Autophagy is a self-protecting cellular catabolic pathway relying on lysosomes. Some long-lived proteins, as well as damaged organelles and misfolded proteins are degraded and recycled through autophagy process (17-19). It is widely acknowledged that autophagy is closely associated with CNS disorders, including cerebral ischemia, Parkinson's disease, and MS (20-23). It has been reported that inducing autophagy could ameliorate several neurodegenerative diseases $(24,25)$. Studies demonstrated that inducing autophagy could inhibit inflammation, especially in inflammatory or immune cells such as macrophages and dendritic cells $(26,27)$. Recently, it has been reported that enhancement of autophagy could ameliorate the pathogenesis of MS or EAE disease through the limit of inflammation $(21,28,29)$. However, whether autophagy plays a role in $\alpha 7 \mathrm{nAChR}$-mediated alleviation of neuroinflammation remains unclear.

In this study, we raised the hypothesis that activation of $\alpha 7 \mathrm{nAChR}$ could promote monocyte/microglia autophagy, which inhibited the production of inflammatory cytokine and thus contributed to the attenuation of EAE severity. Our study may provide a novel therapeutic strategy for the treatment of MS.

\section{MATERIALS AND METHODS}

\section{Animal Care and Use}

C57BL/6J mice (8-10 weeks old, male) were provided by Shanghai Super-B\&K Laboratory Animal Corp. Ltd. (Shanghai, China). Mice were separately housed and had free access to water and standard chow diet (Shanghai Super-B\&K Laboratory Animal Corp. Ltd., Shanghai, China). Experimental mice were maintained in specific pathogen-free conditions under a $12 / 12 \mathrm{~h}$ light cycle (on: 8:00 am) at $23 \pm 2{ }^{\circ} \mathrm{C}$ and $60 \pm 10 \%$ humidity.

\section{BV2 Microglia Culture and Treatment}

Murine BV2 microglia were provided by Shanghai Jining Corp. Ltd. (Shanghai, China). Cells were cultured with DMEM (Gibco, Grand Island, NY, USA) supplemented with 10\% (vol/vol) fetal bovine serum (FBS) (Gibco, Grand Island, NY, USA) at $37^{\circ} \mathrm{C}$ in a humidified incubator with $5 \% \mathrm{CO}_{2}$. BV2 microglia were challenged with lipopolysaccharide (LPS) (Sigma-Aldrich, St. Louis, $\mathrm{MO}, \mathrm{USA}$ ) in a dose of $100 \mathrm{ng} / \mathrm{ml}$ with or without $0.1,1$, and $10 \mu \mathrm{M}$ PNU282987 (Sigma-Aldrich, St. Louis, MO, USA) for $12 \mathrm{~h}$.
In another set of experiments, BV2 microglia were preincubated with compound C (10 $\mu \mathrm{M}$, Sigma-Aldrich, St. Louis, MO, USA), an AMPK inhibitor, for $10 \mathrm{~min}$ before LPS and PNU282987 treatment. For the blockade of autophagy in vitro, bafilomycin A1 (5 nM, Selleckchem, Houston, TX, USA) or autophagy-related gene 5 small-interfering RNA (Atg5 siRNA) were applied for study.

\section{Transient Transfection of Atg5 siRNA}

The following siRNAs against Atg5 (Gene ID: 11793) were synthesized by Genepharm Biotech (Shanghai, China): siRNA1, 5'-CUCUCUAUCAGGAUGAUTT-3', 5' -AUCUCAUCCUGA UAGAGAGTT-3'; siRNA2, 5' -GACGUUGGUAACUGACAAA TT-3' 5' $^{\prime}$-UUUGUCAGUUACCAACGUCTT-3' ; siRNA3 3, 5' -GC GGUUGAGGCUCACUUUATT-3'， 5'-UAAAGUGAGCCUCA ACCGCTT-3'; siRNA4, 5'-GCUACCCAGAUAACUUUCU TT-3', 5'-AGAAAGUUAUCUGGGUAGCTT-3'. All of the four siRNAs comprised 21 nucleotides and contained symmetric $3^{\prime}$ overhangs of two deoxythymidines. BV2 microglia were transfected with Atg5 siRNA as described before $(30,31)$. In brief, murine BV2 microglia were cultured with DMEM supplemented with $10 \%$ (vol/vol) FBS and grown to $30-50 \%$ confluency in sixwell plates for transfection. The control or Atg 5 siRNA ( $5 \mathrm{nM}$ ) was transfected into cells using siRNA-Mate (Genepharm) reagent in the form of siRNA-siRNA-Mate complex. After $24 \mathrm{~h}$ incubation, the medium was changed to fresh $10 \%$ FBS-loaded DMEM for the analysis of protein knockdown or further experiments.

\section{EAE Induction and Assessment}

Mice were carefully grouped and selected randomly for studies. During animal experiments, a design of single-blind study was applied. EAE was induced in mice (8-10 weeks old, male) as previously reported $(32,33)$. In brief, mice were subcutaneously immunized with $200 \mu \mathrm{g} \mathrm{MOG}_{35-55}$ in Complete Freund's adjuvant (Sigma-Aldrich, St. Louis, MO, USA) contained with heat-killed Mycobacterium tuberculosis (H37RA strain, $5 \mathrm{mg} / \mathrm{ml}$ ) (BD Diagnostics, Franklin Lakes, NJ, USA). Pertussis toxin (Calbiochem, Billerica, MA, USA) in a dose of $200 \mathrm{ng}$ for each mouse was injected on days 0 and 2 via i.p. Experimental mice were examined and evaluated every day for clinical signs and were scored according to the following criteria: "0," no clinical signs; “1," paralyzed tail; “2," paresis; “3," paraplegia; “4," paraplegia with forelimb weakness or paralysis; " 5 ," moribund or death. For the treatment of drugs, PNU282987 (0.1 mg/kg, i.p.) or 3-methyladenine (3-MA) (10 mg/kg, i.p.) (Sigma-Aldrich, St. Louis, MO, USA) was injected once a day from day 3 till the end of the study. Mice were treated with saline as vehicle control (100 $\mu$ l for each mouse).

\section{Histopathological Analysis}

Experimental mice were anesthetized with phenobarbital sodium (35 mg/kg, i.p.) and sacrificed by cervical dislocation. Animals were then perfused with PBS ( $\mathrm{pH} 7.4,20 \mathrm{ml}$ ) followed by $4 \%$ (w/v) paraformaldehyde $(20 \mathrm{ml})$. Spinal cords were subsequently excised and further fixed in $4 \%(\mathrm{w} / \mathrm{v})$ paraformaldehyde overnight. Five- $\mu \mathrm{m}$-thick sections of spinal cords embedded with paraffin were stained by hematoxylin and eosin as well as 
luxol fast blue to measure spinal inflammatory infiltration or demyelination, respectively.

\section{Immunofluorescence Microscopy}

Immunofluorescence microscopy of paraffin-embedded sections of spinal cords was performed. In brief, 5 - $\mu$ m-thick paraffinembedded sections of spinal cords were deparaffinized with xylene, rehydrated, blocked with normal goat serum, and incubated with one of the following antibodies: microtubule associated protein 1 light chain (LC) 3 (1:200, Novus Biologicals, Littleton, CO, USA), CD45 (1:200, Abcam, Cambridge, MA, USA), CD68 (1:200, Goodbio, Wuhan, Hubei, China). After extensive washing, the sections were incubated with double immunofluorescent staining including Alexa-488 and Alexa-647-labeled secondary antibodies (1:500, Invitrogen, USA) incubation for $1 \mathrm{~h}$ at room temperature. After being washed, slides were mounted with Vectashield mounting medium containing DAPI (Vector Laboratories, Burlingame, CA, USA) and the colocalization was detected by means of a confocal laser scanning microscope (Fluoview FV1000; Olympus, Tokyo, Japan).

\section{Immunoblot Analysis}

BV2 microglia or spinal cord and spleen from EAE mice were washed with PBS for one time and lysed in lysis buffer on ice for 30 s. Protein concentration was detected by the bicinchoninic acid method (Thermo Scientific, Pittsburgh, PA, USA). Samples were loaded in $10 \%$ or $12 \%$ Tris/Gly gels, subjected to SDSPAGE, and transferred on NC membranes (Millipore, Billerica, MA, USA). Immunoblot was conducted using the rabbit antiBeclin 1 monoclonal antibody (1:500; Cell Signaling Technology, Danvers, MA, USA), rabbit anti-LC3 polyclonal antibody (1:500; Novus Biologicals, Littleton, CO, USA), rabbit anti-p62 antibody (Cell Signaling Technology, Danvers, MA, USA), rabbit antiadenosine 5'-monophosphate (AMP)-activated protein kinase (AMPK) antibody (1:500, Cell Signaling Technology, Danvers, MA, USA), rabbit anti-phosphorylated AMPK antibody (1:500, Cell Signaling Technology, Danvers, MA, USA) anti-mammalian target of rapamycin rabbit (mTOR) antibody (1:500, Cell Signaling Technology, Danvers, MA, USA), rabbit anti-phosphorylated mTOR antibody (1:500, Cell Signaling Technology, Danvers, MA, USA), rabbit anti-p70 ribosomal protein S6 kinase (p70S6K) antibody (1:500, Cell Signaling Technology, Danvers, MA, USA), rabbit anti-phosphorylated p70S6K antibody (1:500, Cell Signaling Technology, Danvers, MA, USA) and mouse anti-glyceraldehyde-3-phosphate dehydrogenase (GAPDH) antibody (1:1000, Beyotime Biotechnology, Shanghai, China). After that, the membranes were incubated with a Donkey antiRabbit or Donkey anti-mouse secondary antibody (1:5,000, LI-COR Biosciences, Lincoln, NE, USA) accordingly. Images were obtained and analyzed using the Odyssey infrared imaging system (LI-COR Bioscience, Lincolin, NE, USA).

\section{Reverse Transcription and Real-time PCR}

TRIzol reagent (Invitrogen, Carlsbad, CA, USA) was used for the extraction of total RNA from BV2 microglia. Reverse transcription was conducted for the extracted RNA to obtain the cDNA with PrimeScript ${ }^{\mathrm{TM}}$ RT Master Mix (Takara, Otsu, Shiga, Japan).
Real-time PCR was then conducted in the LightCycler quantitative PCRapparatus(Stratagene,SantaClara,CA,USA) usingtheFastStart Unitversal SYBR Green Master (Roche, Konzern-Hauptsitz, Grenzacherstrasse, Switzerland). Expression value was normalized to GAPDH in the same sample and then normalized to the control. The sequences of the primer pairs are listed as followed: interleu-

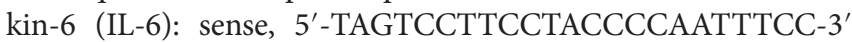
and antisense, 5'-TTGGTCCTTAGCCACTCCTTC-3'; IL-1 $\beta$ : sense, 5'-CTCGTGCTGTCGGACCCCAT-3' and antisense, 5'AGTGTTCGTCTCGTGTTCGGAC-3'; IL-18: sense, 5' - CAGGC CTGACATCTTCTGCAA- ${ }^{\prime}$ and antisense, $5^{\prime}$ - CTCCAGCATCA GGACAAAGAAAGCCG-3'; tumor necrosis factor- $\alpha$ (TNF- $\alpha)$ : sense, 5' - AAGCCTGTAGCCCACGTCGTA- $3^{\prime}$ and antisense, 5'- GGCACCACTAGTTGGTTGTCTTTG-3'; GAPDH: sense, 5'-GTATGACTCCACTCACGGCAAA-3' and antisense, 5' - GGT CTCGCTCCTGGAAGATG-3'.

\section{Transmission Electron Microscopy}

Murine BV2 microglia were cultured at $37^{\circ} \mathrm{C}$ on glass coverslips overnight, followed by the treatments mentioned above. BV2 microglia were harvested and fixed overnight at $4^{\circ} \mathrm{C}$ in $2.5 \%$ glutaraldehyde in $0.1 \mathrm{M} \mathrm{PBS}$, and then post-fixed in $1 \%$ buffered osmium tetroxide for $2 \mathrm{~h}$. Specimens were processed in routine procedure and examined under a transmission electron microscope (H-700; Hitachi, Tokyo, Japan).

\section{Autophagy Flux Assessment}

Murine BV2 microglia were seeded on the cultural slides and transfected with tandem fluorescent mRFP-GFP-LC3 plasmid (HanBio, Shanghai, China) when the confluence reached to $50-70 \%(34,35)$. In brief, after the culture in DMEM supplemented with $10 \%$ (vol/vol) FBS for $24 \mathrm{~h}$, cells were incubated with plasmids for $6 \mathrm{~h}$ and then changed back to fresh DMEM supplemented with $10 \%$ (vol/vol) FBS for the cultivation of another $36 \mathrm{~h}$ to ensure the expression of the genes. After transfection, cells were challenged with $100 \mathrm{ng} / \mathrm{ml}$ LPS for $12 \mathrm{~h}$ in the presence or absence of PNU282987 $(10 \mu \mathrm{M})$ for $10 \mathrm{~min}$. Cellular autophagosomes $\left(\mathrm{G}^{+} \mathrm{R}^{+}\right)$and autolysosomes $\left(\mathrm{G}^{-} \mathrm{R}^{+}\right)$were detected by confocal microscopy (Leica TCS SP8, Leica, Biberach, Germany). Total number of puncta $(>1 \mu \mathrm{m})$ per cell was counted.

\section{Cell Viability Assay}

Cell viability was evaluated using a non-radioactive cell counting kit-8 (CCK-8; Dojindo, Kamimashiki-gun Kumamoto, Japan) as described previously (36). In brief, murine BV2 microglia at the density of $1 \times 10^{4}$ were seeded in a 96-well plate in DMEM supplemented with $10 \%$ (vol/vol) FBS. After cultivation for $6 \mathrm{~h}$, cells were treated as described above. After $12 \mathrm{~h}$ treatment, CCK- 8 culture medium was added for $1 \mathrm{~h}$ additional cultivation. Absorbance was assayed with a microplate reader (Tecan Group Ltd., Männedorf, Switzerland) at the wavelength of $450 \mathrm{~nm}$ for the analysis of cell viability.

\section{Statistics}

Data were presented as mean \pm SEM. A two-way analysis of variance (ANOVA) followed by Bonferroni post hoc test for 
repeated measures was used for the analysis of the statistical significance of the EAE clinical scores between treatments. For other analysis, a Kruskal-Wallis test followed by Dunn's post hoc test and one-way ANOVA followed by Bonferroni post hoc test were used to determine non-parametric data and continuous variables, respectively. A $P$-value $<0.05$ was considered statistically significant. Data were analyzed with SPSS $21.0 \mathrm{~K}$ for Windows (SPSS, Chicago, IL, USA).

\section{RESULTS}

\section{Activating $\alpha 7 \mathrm{nAChR}$ Ameliorates EAE Severity and Spinal Inflammatory Infiltration in EAE}

To assess the role of $\alpha 7 \mathrm{nAChR}$ on CNS disorders, we treated EAE mice with $\alpha 7 \mathrm{nAChR}$ agonist PNU282987 in the doses of 0.03 and $0.1 \mathrm{mg} / \mathrm{kg}$ body weight, respectively, from days
3 postimmunization (PI) till the end of study, and found that PNU282987 at the dose of $0.1 \mathrm{mg} / \mathrm{kg}$ body weight could effectively reduce the peak severity and cumulative clinical score of EAE while only slight decreases in the peak severity and cumulative clinical score were detected at the dose of $0.03 \mathrm{mg} / \mathrm{kg}$ body weight (Figure 1A). Histological study of spinal cord was performed at day 17 PI. Compared with vehicle, PNU282987 $(0.1 \mathrm{mg} / \mathrm{kg})$ caused a significant reduction of leukocyte infiltration and alleviative demyelination in spinal cord shown by $\mathrm{H} \& \mathrm{E}$ and luxol fast blue staining (Figures 1B,C).

\section{Activating $\alpha 7 n A C h R$ Increases Autophagy in Spinal Cord and Spleen from EAE Mice}

It has been previously demonstrated that autophagy plays a protective and ameliorative role in EAE through the regulation of inflammatory cytokine production (21). We thus ask whether the protective effect of activating $\alpha 7 \mathrm{nAChR}$ on EAE is associated with the augmented autophagy. First, we tested the
A

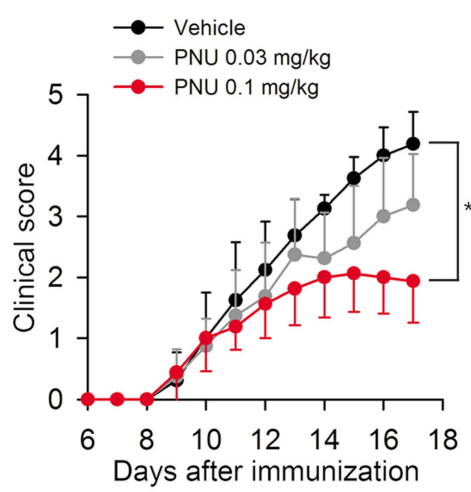

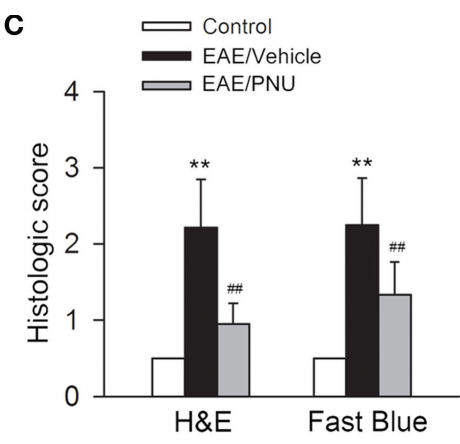

C H\&E Fast Blue
B
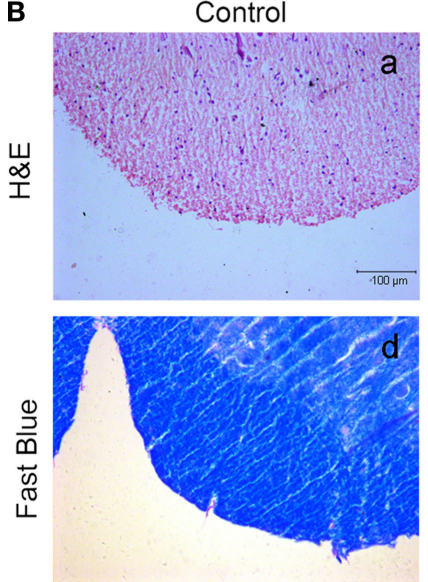

Vehicle
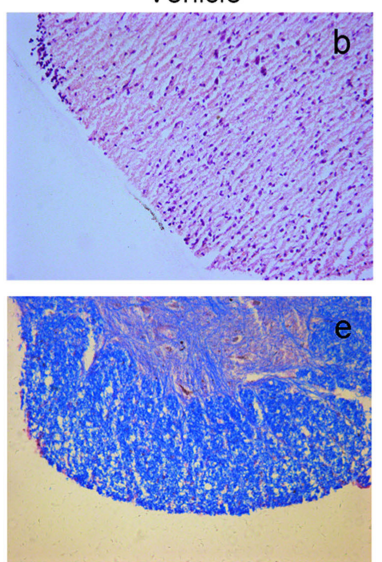

PNU

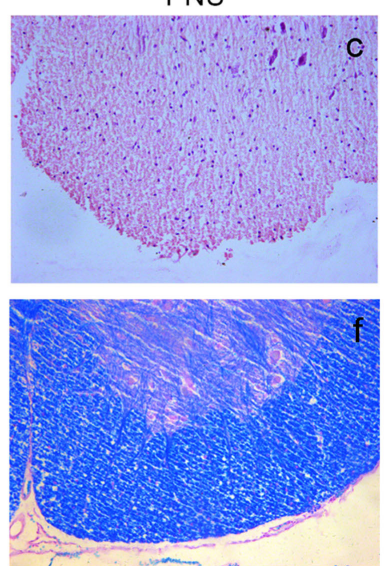

FIGURE $1 \mid \alpha 7 n A C h R$ activation alleviates experimental autoimmune encephalomyelitis (EAE) severity and central nervous system inflammatory infiltration in EAE mice. EAE mouse model was created and treated with PNU282987 (0.03 and 0.1 mg/kg, i.p.) or vehicle from day 3 postimmunization and were maintained on drug for the duration of the study. (A) Clinical signs were assessed daily. $0.1 \mathrm{mg} / \mathrm{kg}$ PNU282987 effectively reduced the peak severity and cumulative clinical score of EAE mice while only slight decreases in the peak severity and cumulative clinical score were detected at the dose of $0.03 \mathrm{mg} / \mathrm{kg}$ body weight $(n=8$ per group). ${ }^{\star} P<0.05$ vs vehicle. (B,C) H\&E and luxol fast blue staining of paraffin sections of spinal cords isolated from control, vehicle, or $\mathrm{PNU} 282987$ (0.1 $\mathrm{mg} / \mathrm{kg}$ )treated EAE mice on day 17 ( $n=8$ per group). PNU282987 ameliorated spinal inflammatory infiltration and demyelination compared with vehicle. Scale bar, 100 mm. ${ }^{\star \star} P<0.01$ vs control; ${ }^{\#} P<0.01$ vs vehicle. Veh, vehicle; PNU, PNU282987. 
effect of activating $\alpha 7 \mathrm{nAChR}$ on the levels of autophagy-related proteins in spinal cord and spleen from EAE mice and found that activating $\alpha 7 \mathrm{nAChR}$ with PNU282987 significantly increased the
LC3-II/I ratio and Beclin 1 abundance and decreased the p62/ SQSTM1 abundance in spinal cord and spleen from EAE mice (Figures 2A,B).
A

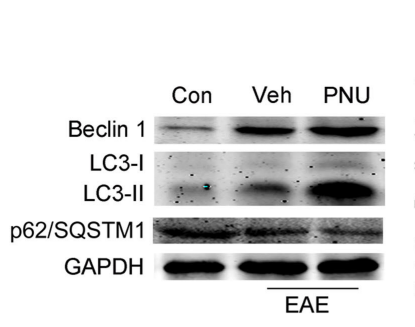

B
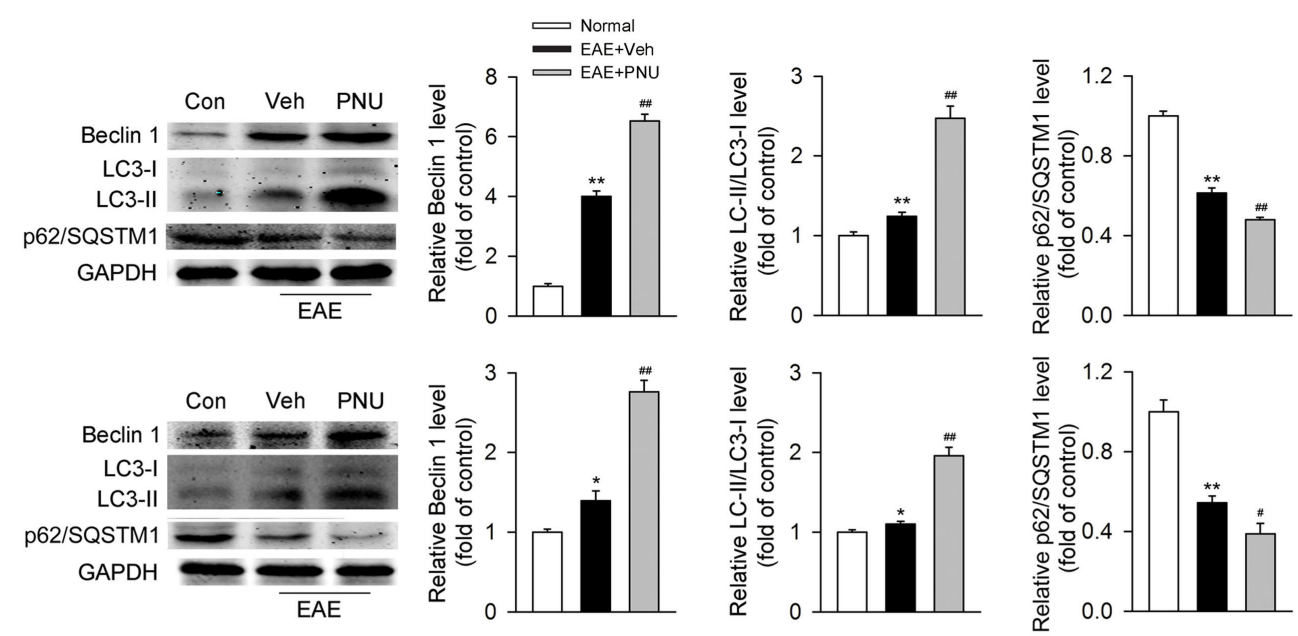

C

CD45

LC3

DAPI

Merge

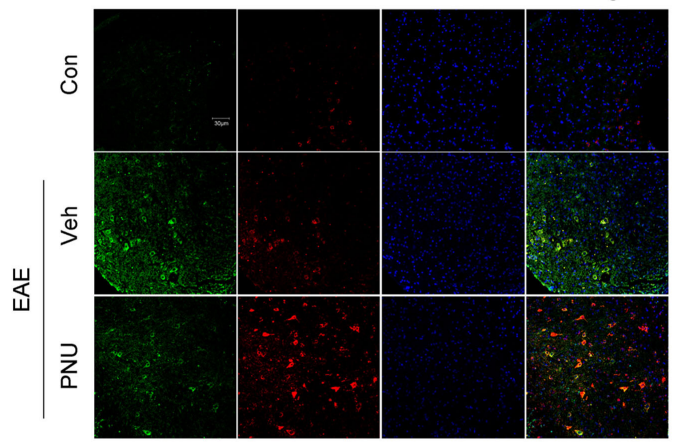

D

\section{ปั}

CD45

LC3

DAPI

Merge

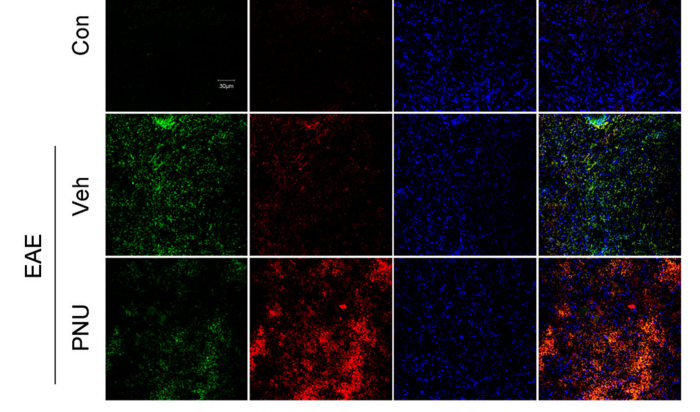

E

CD68

LC3

DAPI

Merge

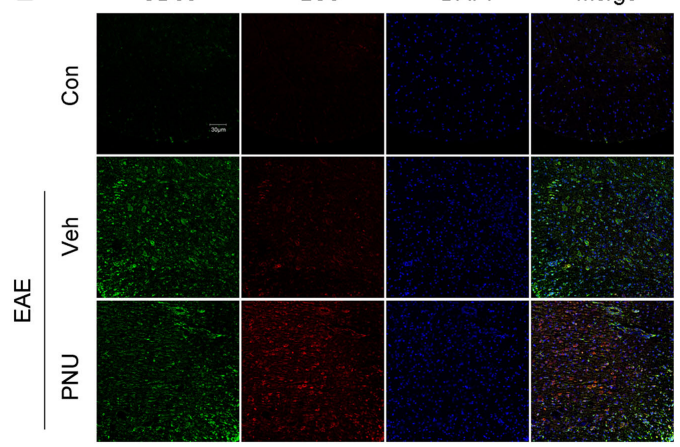

F

F
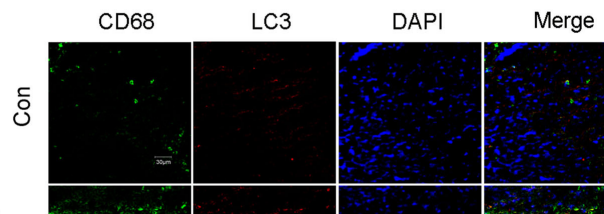

崫
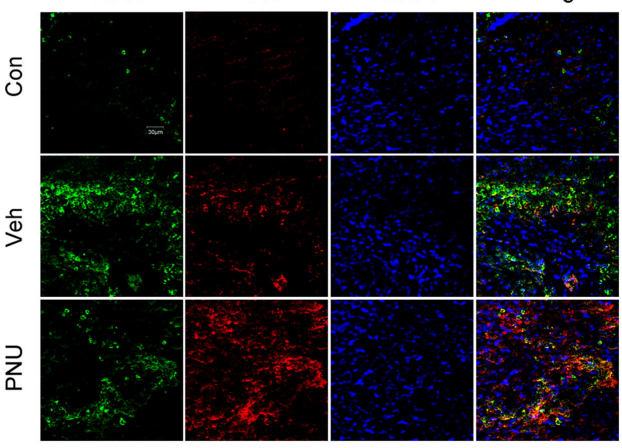

FIGURE $2 \mid \alpha 7 n A C h R$ activation leads to the enhancement of monocyte/macrophage autophagy in spinal cord and spleen from experimental autoimmune encephalomyelitis (EAE) mice. EAE mouse model was created and treated with PNU282987 (0.1 mg/kg, i.p.) or vehicle from day 3 postimmunization $(\mathrm{PI})$ and were maintained on drug for the duration of the study. (A,B) Spinal cord and spleen were isolated from mice on day $17 \mathrm{PI}$, and then lysed with buffer. (A) Levels of autophagy-related proteins were detected by Western blotting in spinal cord. PNU282987-treated EAE mice significantly enhanced the levels of Beclin 1 and LC3-II/I ratio and reduced the level of p62/SQSTM1 compared with vehicle ( $n=6$ per group). Quantitative analysis was conducted for the relative levels of Beclin 1, LC3-II/I ratio, and p62/SQSTM1. ${ }^{*} p<0.01$ vs Veh. (B) The same detections were conducted for the levels in spleen $\left(n=6\right.$ per group). ${ }^{*} P<0.05$ vs control; ${ }^{* *} P<0.01$ vs control; ${ }^{\#} P<0.05$ vs vehicle; ${ }^{\#} P<0.01$ vs vehicle. (C) Representative images of immunofluorescence microscopy of spinal cord paraffinembedded sections for the staining of CD45, LC3, and DAPI. Scale bar, $30 \mu \mathrm{m}$. (D) Representative images of immunofluorescence microscopy of spleen paraffinembedded sections for the staining of CD45, LC3, and DAPI. Scale bar, $30 \mu \mathrm{m}$. (E) Representative images of immunofluorescence microscopy of spinal cord paraffin-embedded sections for the staining of CD68, LC3, and DAPI. Scale bar, $30 \mu \mathrm{m}$. (F) Representative images of immunofluorescence microscopy of spleen paraffin-embedded sections for the staining of CD68, LC3, and DAPI. Scale bar, $30 \mu \mathrm{m}$. Veh, vehicle; PNU, PNU282987. 
Second, to determine the change of monocyte autophagy including macrophages in periphery and microglia in central (hereafter referred to as "monocyte/microglia autophagy") upon activation of $\alpha 7 \mathrm{nAChR}$, we tested the colocalization of CD45- or CD68-positive cells with LC3, respectively, in spinal cord and spleen from the control and EAE mice with or without the treatment of PNU282987. We found that compared with the control group, EAE mice had more colocalization of CD45or CD68-positive cells with LC3 in spinal cord and spleen. PNU282987 treatment further increased the colocalization of CD45 (Figures 2C,D) or CD68 (Figures 2E,F) with LC3, suggesting that activating $\alpha 7 \mathrm{nAChR}$ contributed to the enhancement of monocyte/microglia autophagy in spinal cord and spleen from EAE mice.

\section{Blockade of Autophagy Attenuates the Protective Effects of $\alpha 7 n A C h R$ Activation on EAE Mice}

To determine the influence of autophagy on the $\alpha 7 \mathrm{nAChR}$ mediated protective effects on the MS course in vivo, we examined neurobehavioral deficits in 3-MA, PNU282987, 3-MA + PNU282987, and vehicle-treated mice following $\mathrm{MOG}_{35-55}$-induction of EAE. As shown in Figure 1, the disease course in the EAE mice model was a chronic progressiverelapsing phenotype. PNU282987 (0.1 mg/kg, i.p.) treatment significantly reduced the severity of neurobehavioral deficits, cumulative scores, and maximum neurological disability in EAE mice compared with these vehicle-treated mice. This protective effect of PNU282987 was abolished by 3-MA (10 mg/kg), an autophagy inhibitor (Figures 3A-C), suggesting that autophagy at least partly played a role in the protective effects of activating $\alpha 7 \mathrm{nAChR}$ on EAE.

As shown above, in EAE mice, the protective effects of PNU282987 treatment was associated with reduced inflammatory
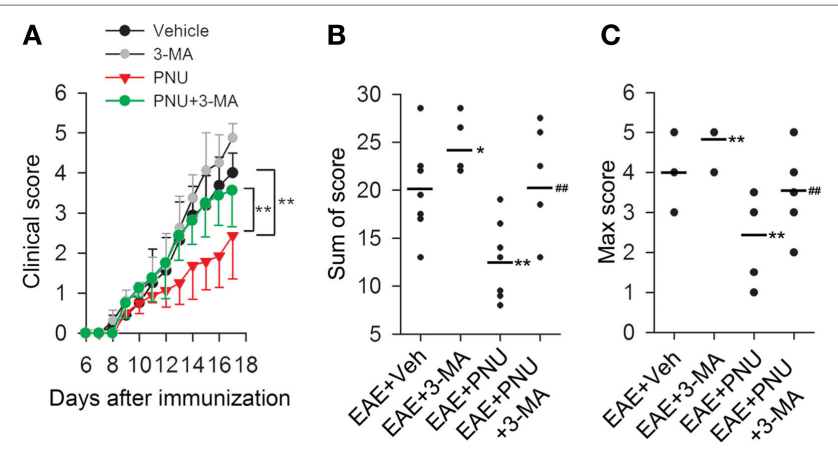

FIGURE 3 | Blockade of autophagy with 3-methyladenine (3-MA) attenuates the alleviative effects of $\alpha 7 \mathrm{nAChR}$ activation on severity in experimental autoimmune encephalomyelitis (EAE) mice. EAE mouse models were created. PNU282987 (0.1 mg/kg, i.p.) was given for the activation of $\alpha 7 \mathrm{nAChR}$ and 3-MA (10 mg/kg, i.p.) was given for the blockade of autophagy process in vivo. The clinical score (A), sum of score (B), and max score (C) were assessed for the analysis of the extent of EAE severity ( $n=8$ per group). ${ }^{*} P<0.05$ vs Veh, ${ }^{* \star} P<0.01$ vs Veh, ${ }^{\#} P<0.01$ vs $P N U$. Veh, vehicle; PNU, PNU282987. infiltration and demyelination. We then tested if 3-MA abolished the protective effects of PNU282987 by upregulating inflammatory infiltration and demyelination. We found that blockade of autophagy with 3-MA greatly attenuated the alleviative effects of PNU282987 on inflammatory infiltration and demyelination (Figures 4A,B). We further investigated the association between autophagy and $\alpha 7 \mathrm{nAChR}$-mediated anti-inflammatory effects in vivo by examining the production of IL- 6 , IL- $1 \beta$, IL-18, and TNF- $\alpha$ in mRNA level in spinal cord and spleen obtained from mice. In EAE mouse model, the treatment of PNU282987 significantly decreased the mRNA levels of IL-6, IL-1 $\beta$, IL-18, and TNF- $\alpha$ in spinal cord and spleen, while blockade of autophagy with 3-MA greatly abolished this effect (Figures 4C,D). Taken together, these data indicated that blockade of autophagy with 3 -MA greatly attenuated the protective effect of $\alpha 7 \mathrm{nAChR}$ activation on the alleviation of EAE symptoms and inhibition of inflammation in EAE mice.

\section{Activating $\alpha 7 n A C h R$ Increases Autophagy in BV2 Microglia Stimulated with LPS}

Upon LPS stimulation, the levels of LC3-II/I ratio and Beclin 1 abundance were significantly increased in BV2 microglia. Whereas p62/SQSTM1, a cargo receptor targeting the substrates into forming autophagosomes, was significantly decreased (Figures 5A,B). Preincubation with PNU282987 (0.1, 1, and $10 \mu \mathrm{M})$ dose-dependently enhanced these LPS-induced effects in BV2 microglia (Figures 5A,B). Since autophagosomes are basic functional units for autophagy, here we further detected the number of autophagosomes in vitro. Under the stimulation of LPS, the number of autophagosomes was greatly increased which was detected by transmission electron microscopy. In addition, PNU282987 $(10 \mu \mathrm{M})$ treatment further enhanced this effect (Figures 5C,D).

\section{Activating $\alpha 7 n A C h R$ Promotes the Level of Autophagy Flux in BV2 Microglia Stimulated with LPS}

Autophagy is considered as a recycling process that includes the maturation of autophagosomes and subsequently the fusion of autophagosomes and lysosomes for the formation of the degradative autolysosomes. Autophagy flux depicts this entire dynamic process. In vitro, the autophagy flux was previously reported to be detected by the transfection of adenovirus harboring mRFPGFP-LC3 (35). After transfection, autophagosomes were shown as yellow punta (the combination of red and green fluorescence), and autolysosomes were shown as red punta (the extinction of GFP in the acid environment of lysosomes). As shown in Figures 6A-C, LPS challenge increased both the number of yellow autophagosomes and red autolysosomes (the extinction of GFP in the acid environment of lysosomes). Preincubation with PNU282987 further enhanced this effect induced by LPS, suggesting that activating $\alpha 7 \mathrm{nAChR}$ increased the conversion from autophagosomes to autolysosomes and induced a high level of autophagy flux. 

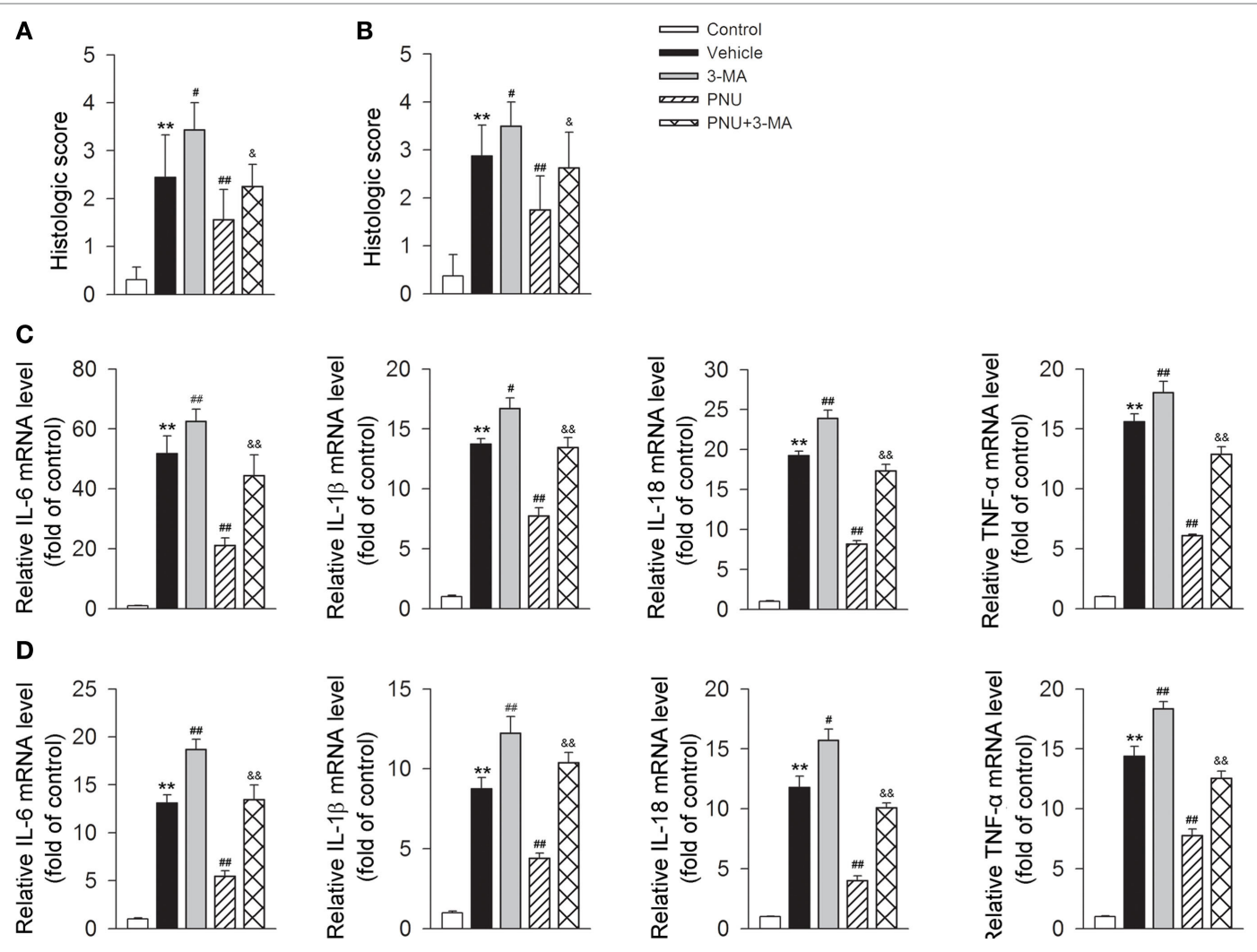

FIGURE 4 | Blockade of autophagy using 3-methyladenine (3-MA) attenuates the alleviative effects of $\alpha 7 n A C h R$ activation on severity and inflammatory response in experimental autoimmune encephalomyelitis (EAE) mice. EAE mouse model was created. PNU282987 (0.1 mg/kg, i.p.) was given for the activation of $\alpha 7 \mathrm{nAChR}$ and 3-MA (10 mg/kg, i.p.) was given for the blockade of autophagy process in vivo. Spinal cord and spleen were isolated from mice on day 17 postimmunization. (A) Quantitative analysis of hematoxylin and eosin staining for the testing of the extent of spinal inflammatory infiltration ( $n=8$ per group). ${ }^{* \star} P<0.01$ vs control, ${ }^{\#} P<0.05$ vs Veh, ${ }^{\# \#} P<0.01$ vs Veh, ${ }^{8} P<0.05$ vs PNU. (B) Quantitative analysis of luxol fast blue staining for the testing of the extent of spinal demyelination ( $n=8$ per group). ${ }^{\star \star} P<0.01$ vs control, ${ }^{\#} P<0.01$ vs Veh, ${ }^{\# \#} P<0.01$ vs Veh, ${ }^{\circledR} P<0.05$ vs PNU. Production of IL-6, IL-1 $\beta$, IL-18, and tumor necrosis factor- $\alpha(\mathrm{TNF}-\alpha)$ in spinal cord (C) and spleen tissue (D) in mRNA level was detected by real-time PCR. The blockade of autophagy process with 3-MA significantly increased the production of IL-6, IL-1 $\beta, \mathrm{IL}-18$, and TNF- $\alpha$ in mRNA level ( $n=6$ per group). ${ }^{\star \star} P<0.01$ vs control, ${ }^{\#} P<0.05$ vs Veh, ${ }^{\# \#} P<0.01$ vs Veh, ${ }^{\text {\& }} P<0.01$ vs PNU. Veh, vehicle; PNU, PNU282987.

\section{Blockade of Autophagy by Atg5 siRNA or Bafilomycin A1 Greatly Attenuates the Anti-inflammatory Effect of PNU282987 in BV2 Microglia Stimulated with LPS}

Upon LPS stimulation, mRNA levels of IL-6, IL-1 $\beta$, IL-18, and TNF- $\alpha$ in BV2 microglia were significantly increased. PNU282987 treatment significantly decreased the mRNA levels of inflammatory cytokines (IL-6, IL-1 $\beta$, IL-18, and TNF- $\alpha$ ). Atg 5 , an E3 ubiquitin ligase, is necessary for autophagy due to its role in autophagosome elongation. Knockdown of Atg5 using Atg5 siRNA (siRNA1 according to Figure 7A) significantly attenuated the inhibitory effect of PNU282987 on the levels of IL-6, IL-1 $\beta$, IL-18, and TNF- $\alpha$ in mRNA (Figures 7B-E). Similar changes were found in the mRNA levels of IL-6, IL-1 $\beta$, IL-18, and TNF- $\alpha$ with the application of bafilomycin A1 (Figure S1A in Supplementary Material). In addition, bafilomycin A1 also significantly inhibited the effect of PNU282987 on LC3-II/I ratio in LPS-stimulated BV2 microglia (Figures S1B,C in Supplementary Material). The administration of LPS, PNU282987, or bafilomycin A1 did not produce significant effect on cell viability (Figure S1D in Supplementary Material). Collectively, those results suggested that autophagy at least partly mediated the anti-inflammatory effect of PNU282987 in LPS-stimulated BV2 microglia.

\section{Participation of the AMPK-mTOR-p70S6K in the Protective Effects of PNU282987 in BV2 Microglia Stimulated with LPS}

It has been previously demonstrated that AMPK activation inhibits mTOR and reduces p70S6K phosphorylation, thus promoting autophagy $(37,38)$. Here, we investigated whether the autophagy-inducing effect of activating $\alpha 7 \mathrm{nAChR}$ was through the AMPK-mTOR-p70S6K signaling pathway in LPS-stimulated BV2 microglia. We found that LPS stimulation increased the phosphorylation of AMPK while decreased the phosphorylation of mTOR and p70S6K. PNU282987 treatment further increased the changes of phosphorylation of AMPK, mTOR, and p70S6K (Figures 8A-D). Furthermore, compound C (an AMPK inhibitor) significantly inhibited the PNU29287-mediated attenuation of 


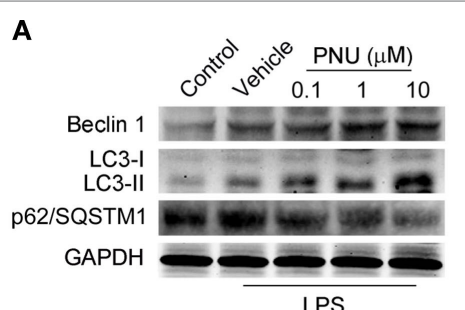

C
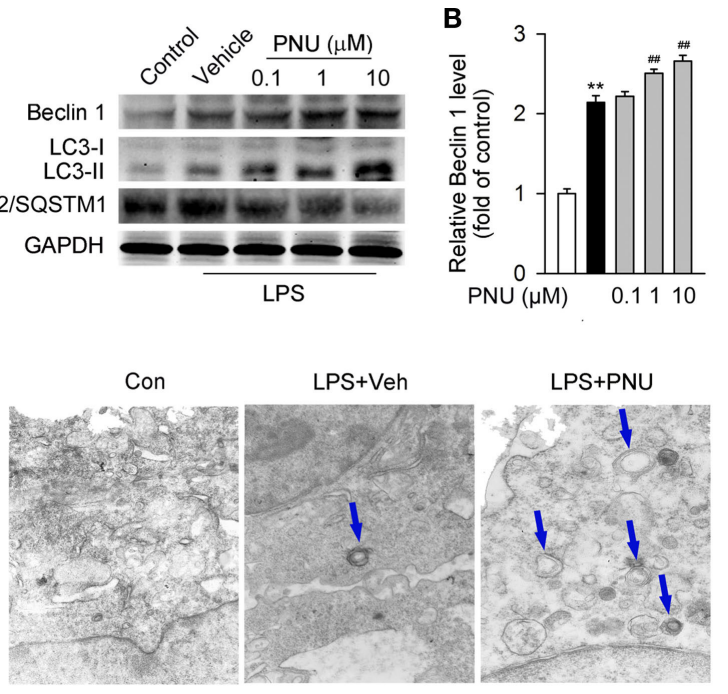
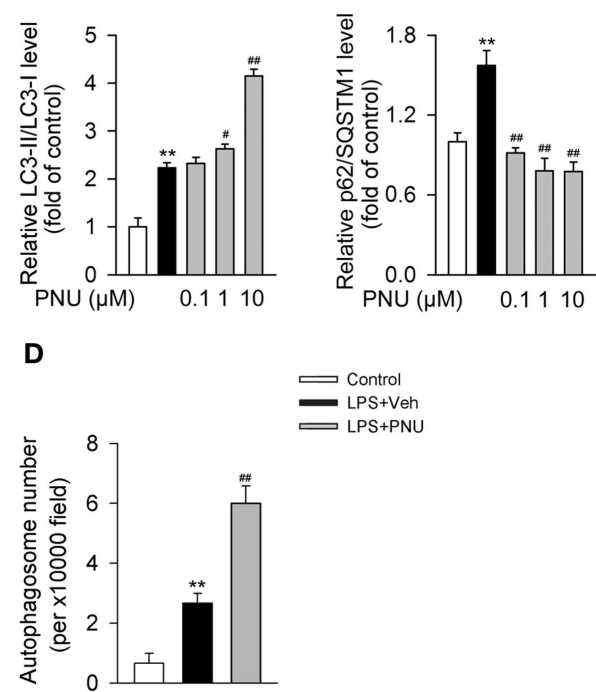

FIGURE 5 | $\alpha$ 7nAChR activation leads to the enhancement of autophagy in BV2 microglia stimulated with lipopolysaccharide (LPS). (A) BV2 microglia were pretreated with vehicle or PNU282987 in three different doses $(0.1,1$, and $10 \mu \mathrm{M})$ and then challenged with LPS (100 ng/ml). Relative expressions of autophagy-related proteins were detected by Western blotting. PNU282987 significantly enhanced the levels of Beclin 1 and LC3-II/I ratio and reduced the expression of p62/SQSTM1 compared with vehicle ( $n=6$ per group). (B) Quantitative analysis of relative levels of Beclin 1, LC3-II/I ratio, and p62/SQSTM1. ${ }^{\star \star} P<0.01$ vs normal, ${ }^{\#} P<0.05$ vs Veh, ${ }^{\#} P<0.01$ vs Veh. (C) BV2 microglia were pretreated with vehicle or PNU282987 (10 $\mu M$ ) and then challenged with LPS (100 ng/ml). The number of autophagosomes was detected by transmission electron microscopy and autophagosomes were marked by blue arrows. PNU282987 significantly increased the number of autophagosomes compared with vehicle ( $n=6$ per group). Magnification, $10,000 \times$. (D) Quantitative analysis of the number of autophagosomes in BV2 microglia. ${ }^{\star \star} P<0.01$ vs normal, ${ }^{\# \#} P<0.01$ vs Veh. Veh, vehicle; PNU, PNU282987.

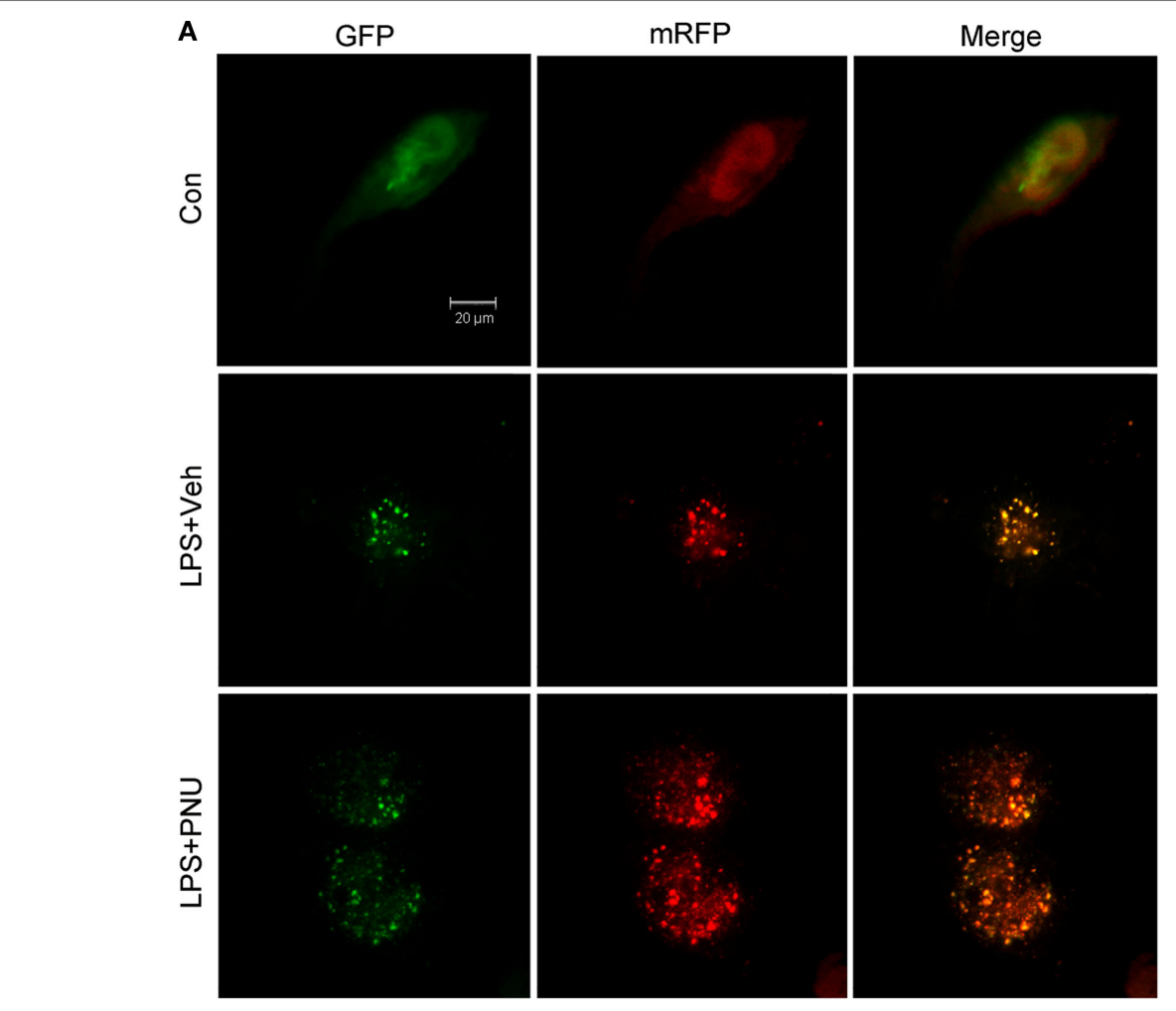

B
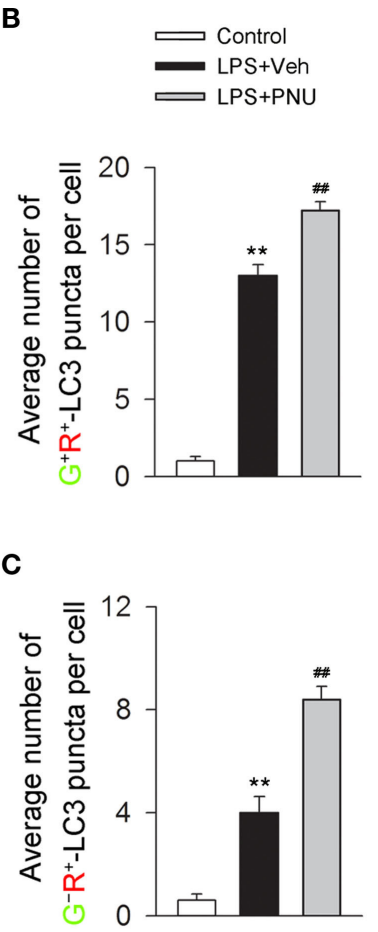

FIGURE 6 | $\alpha 7 n$ AChR activation increases the level of autophagy flux in BV2 microglia stimulated with lipopolysaccharide (LPS). (A) BV2 microglia were pretreated with vehicle or PNU282987 (10 $\mu \mathrm{M})$ and representative images of mRFP-GFP-LC3 vector were shown by fluorescent detection. PNU282987 significantly increased the level of autophagy compared with vehicle ( $n=5$ per group). Scale bar, $20 \mu \mathrm{m}$. (B,C) Quantitative analysis of the number of yellow autophagosomes and red autolysosomes. ${ }^{\star \star} P<0.01$ vs normal, ${ }^{\# \# P}<0.01$ vs Veh. Veh, vehicle; PNU, PNU282987. 

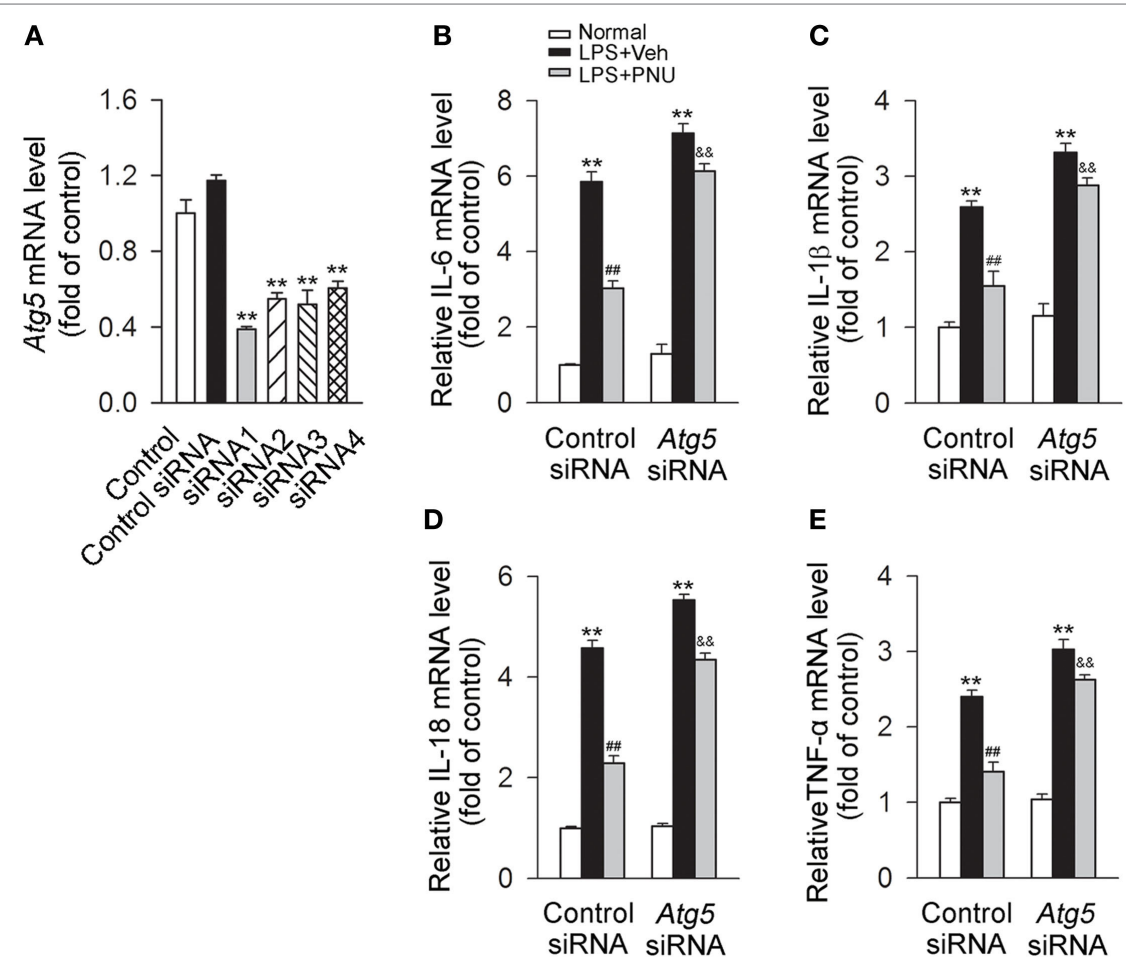

FIGURE 7 | Blockade of autophagy by Atg5 siRNA greatly attenuates the anti-inflammatory effect of PNU282987 in BV2 microglia stimulated with lipopolysaccharide (LPS). (A) The quantitative expression of Atg5 mRNA in BV2 microglia after transfection with the control siRNA or Atg5 siRNAs. ** $>0.01$ vs the control siRNA. After transfection with the control siRNA or Atg5 siRNA [Atg5 siRNA1 according to panel (A)], BV2 microglia were pretreated with vehicle or PNU282987 (10 $\mu \mathrm{M})$ and then challenged with LPS (100 ng/ml). The control or Atg5 siRNA were transfected ( $n=6$ per group). Production of interleukin-6 (IL-6) (B), IL-1 $\beta$ (C), IL-18 (D), and tumor necrosis factor- $\alpha(T N F-\alpha)$ (E) in mRNA level were detected by real-time PCR. The blockade of autophagy process with Atg5 siRNA significantly increased the production of IL-6, IL-1 $\beta, I L-18$ and TNF- $\alpha$ in mRNA level ( $n=6$ per group). ${ }^{\star \star} P<0.01$ vs normal, ${ }^{\# \#} P<0.01$ vs Veh, ${ }^{\text {\&\& }} P<0.01$ vs control. Veh, vehicle; PNU, PNU282987.

IL-6, IL-1 $\beta$, IL-18, and TNF- $\alpha$ production in LPS-stimulated BV2 microglia (Figures 8E-H). Taken together, these data indicated that the anti-inflammatory effects mediated by $\alpha 7 \mathrm{nAChR}$ via inducing autophagy were at least partly through the AMPKmTOR-p70S6K signaling pathway.

\section{DISCUSSION}

Our previous works have demonstrated that activating $\alpha 7 \mathrm{nAChR}$ plays a protective role in several kinds of cardiovascular diseases including ischemic stroke, hypertension, and myocardial ischemia via the "cholinergic anti-inflammatory pathway." It has been demonstrated previously that activating $\alpha 7 \mathrm{nAChR}$ alleviates EAE through the inhibition of inflammatory reaction (14-16). For example, Hao et al. (16) demonstrated that $\alpha 7 \mathrm{nAChR}$ was a key mediator in the process of nicotine-mediated reduction of the CNS inflammatory response and protection against EAE through the inhibition of auto-reactive T-cell proliferation and cytokine production from helper $\mathrm{T}$ cell. Consistent with those reports, in our present study, we demonstrated that activating $\alpha 7 \mathrm{nAChR}$ significantly reduce the severity in clinical score and histological examination. The production of several inflammatory cytokines including IL-6, IL-1 $\beta$, IL-18, and TNF- $\alpha$ was attenuated with the activation of $\alpha 7 \mathrm{nAChR}$ in EAE mice. In combination, those data indicate that activating $\alpha 7 \mathrm{nAChR}$ contributes greatly to the alleviation of EAE.

Autophagy has been recognized as a self-protective mechanism through degrading and recycling long-lived proteins, damaged organelles and misfolded proteins (39). Previous studies demonstrated that autophagy played an important role in neuroprotection through the modulation of inflammatory or immune reaction in CNS in MS or EAE $(6,40-43)$. For instance, it has been demonstrated (42) that the mammalian target of rapamycin (mTOR), a negative regulator of autophagy, was involved in microglial pro-inflammatory activation. Rapamycin, an mTOR inhibitor, ameliorated the clinical course of the relapsing-remitting as well as the chronic EAE through the induction of microglia autophagy. Autophagy level has also been demonstrated to be enhanced in EAE mice $(34,42)$. In our previous work, we demonstrated that activation of cannabinoid receptor 2 could induce autophagy, which subsequently led to the inhibition of NLRP3 inflammasome in CNS in EAE as well as colitis mice models $(6,44)$. Here, we reported that blocking autophagy with 3-MA could attenuate the anti-inflammatory and alleviative effects mediated by $\alpha 7 \mathrm{nAChR}$ activation in EAE mice. Similar results were found in LPS-stimulated BV2 microglia when transfected with Atg5 siRNA or the application of bafilomycin A1. Taken together, those data indicate that autophagy plays an important 

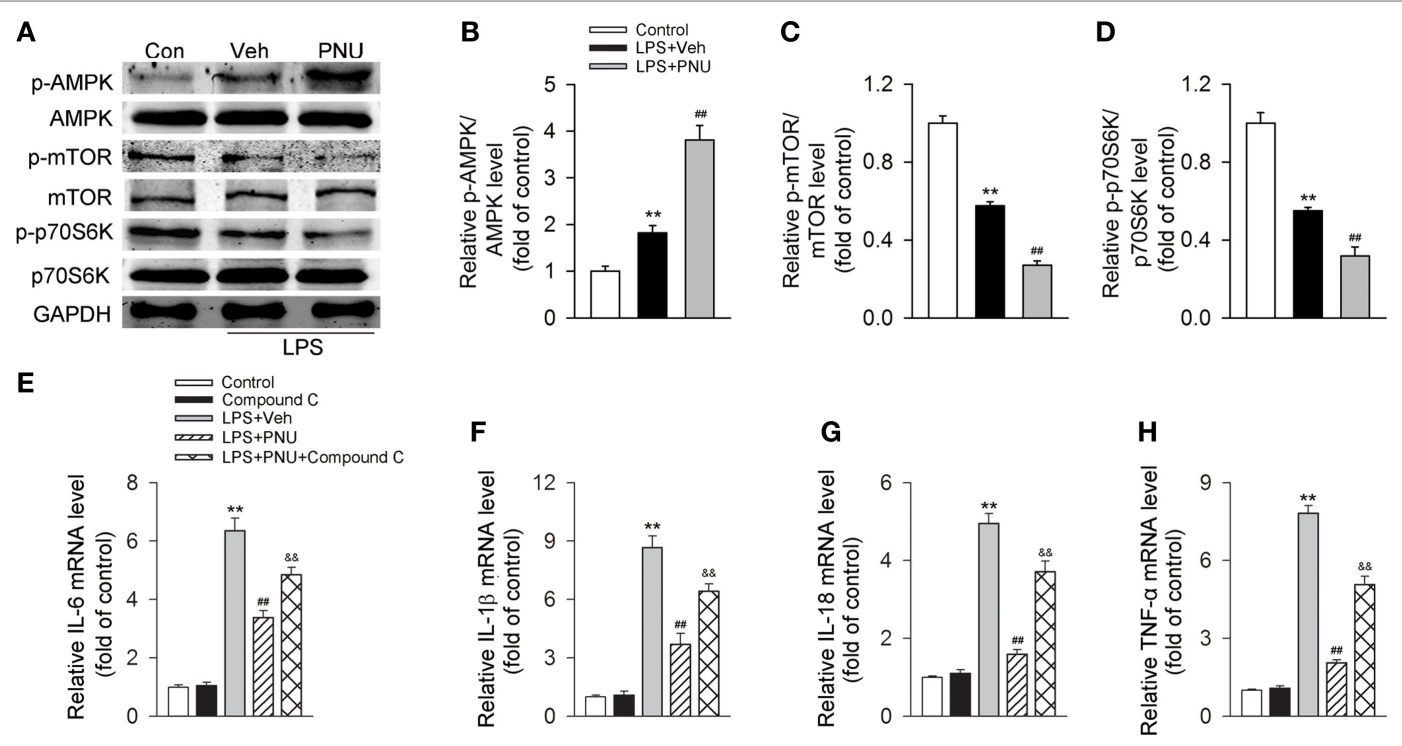

FIGURE 8 | Participation of the AMPK-mTOR-p70S6K axis in the anti-inflammatory effect of PNU282987 in BV2 microglia stimulated with lipopolysaccharide (LPS). BV2 microglia were pretreated with vehicle or PNU282987 (10 $\mu$ M) and then challenged with LPS (100 ng/ml). (A) Levels of p-AMPK/ AMPK, p-mTOR/mTOR, and p-p70S6K/p70S6K ratios were detected by Western blotting. PNU282987 significantly enhanced the ratios of p-AMPK/AMPK, $\mathrm{p}-\mathrm{mTOR} / \mathrm{mTOR}$, and p-p70S6K/p70S6K compared with vehicle ( $n=6$ per group). (B-D) Quantitative analysis of relative levels of p-AMPK/AMPK, p-mTOR/mTOR, and p-p70S6K/p70S6K ratios. ${ }^{* *} P<0.01$ vs normal, ${ }^{\# \#} P<0.01$ vs Veh. (E-H) Production of interleukin-6 (IL-6), IL-1 $\beta$, IL-18, and tumor necrosis factor- $\alpha$ (TNF- $\alpha$ ) in mRNA level were detected by real-time PCR. The blockade of the AMPK-mTOR-p70S6K signaling pathway with compound $\mathrm{C}$ (10 $\mu \mathrm{M}$ ) significantly increased the production of IL-6, IL-1 $\beta$, IL-18, and TNF- $\alpha$ in mRNA level ( $n=6$ per group). ${ }^{*} P<0.01$ vs normal, ${ }^{\# \#} P<0.01$ vs Veh. Veh, vehicle; PNU, PNU282987.

role in the amelioration of EAE as well as the anti-inflammatory effects in LPS-challenged BV2 microglia mediated by the activation of $\alpha 7 \mathrm{nAChR}$.

Recently, activating $\alpha 7 \mathrm{nAChR}$ has been reported to play a protective role in CNS inflammation. The association between activating $\alpha 7 \mathrm{nAChR}$ and induction of autophagy in neurons has been demonstrated. Jeong and Park reported that melatonin, a secretory hormone produced from various organs, enhanced the activation of autophagy process in neurons through the upregulation of $\alpha 7 \mathrm{nAChR}$ signaling pathway, thus playing an important role in neuroprotection in prion-mediated neurodegenerative diseases (45). In their other article, the authors showed that the activation of $\alpha 7 \mathrm{nAChR}$ stimulated by cellular prion protein expression contributed to the induction of autophagy flux in neurons, which played a pivotal role in neuroprotection (46). Their studies suggest an important role of $\alpha 7 \mathrm{nAChR}$ - induce autophagy, which play a protective role in neuroprotection. Here, in our work, we demonstrated that activating $\alpha 7 \mathrm{nAChR}$ contributed to the enhancement of autophagy both in spinal cord and spleen from EAE mice and BV2 microglia stimulated with LPS, and blockade of autophagy greatly attenuated its anti-inflammatory effect. Collectively, we believe that activating $\alpha 7 \mathrm{nAChR}$ may suppress the inflammatory reaction through the induction of monocyte/microglia autophagy, thus playing an important role in neuroprotection as well as alleviation of EAE. Together with the recently proved neuroprotective effects of $\alpha 7 \mathrm{nAChR}$-mediated autophagy in neurons, we believe that enhancing $\alpha 7 \mathrm{nAChR}$-mediated autophagy may probably serve as a potential and promising therapeutic strategy in the treatment of CNS diseases.
In the in vivo studies, we used 3-MA to inhibit autophagy process for the detection of autophagy participation in the pathogenesis and progression of EAE, since 3-MA was widely used as an autophagy blocker $(20,47)$. Our results showed that 3-MA significantly attenuated the anti-inflammatory effects of activating $\alpha 7 \mathrm{nAChR}$ in EAE model, thus demonstrating the involvement of $\alpha 7 \mathrm{nAChR}$-mediated autophagy in EAE disease. However, as discussed previously, the application of 3-MA as an autophagy blocker was not without its problems. It was reported that 3-MA could inhibit all clarifications of PtdIns $3 \mathrm{~K}$ and the related downstream signaling cascades, indicating that its specificity as an autophagy suppressor should be carefully considered $(6,20)$. It was further noted that the long-term application of 3-MA might slightly enhance autophagy level since 3-MA was not a satisfactory autophagy-specific inhibitor (48). In consideration of those limitations of 3-MA, Atg5 siRNA was applied in the present study, since Atg5 was regarded as a critical and necessary autophagyrelated gene, participating the formation of autophagosome (49). We found that Atg5 siRNA or the application of bafilomycin A1 significantly attenuated the inhibitory effect of PNU282987 on the mRNA levels of IL- 6 , IL-1 $\beta$, IL-18, and TNF- $\alpha$ in BV2 microglia, indicating that autophagy at least partly mediated the anti-inflammatory effects of PNU282987 in LPS-stimulated BV2 microglia. According to those discussions, to further clarify the exact role of monoctye/microglia autophagy in EAE, other different kinds of autophagy inhibitors are demanded and more detailed works are warranted.

In this study, we demonstrated that the induction of autophagy by activating $\alpha 7 \mathrm{nAChR}$ could produce the anti-inflammatory 
effects in microglia, which were at least partly through the AMPK-mTOR-p70S6K signaling pathway. However, previous studies have shown other signaling cascades involved in the $\alpha 7 \mathrm{nACh}$-mediated anti-inflammation (50-54). For example, it was reported that $\alpha 7 \mathrm{nAChR}$ could regulate the immune and inflammatory reaction via PI3K-Akt-mTOR signaling cascade (52). Studies from our lab and others that JAK2-STAT3 cascade was involved in the anti-inflammatory effect induced by $\alpha 7 \mathrm{nAChR}$ $(53,54)$. In addition, $\mathrm{p} 38 /$ Src-dependent pathway was considered to participate in the $\alpha 7 \mathrm{nAChR}$-mediated anti-inflammatory effects $(50,51)$. Based on those studies, we believe that the anti-inflammatory effects mediated by $\alpha 7 \mathrm{nAChR}$ probably involve several signaling pathways. Together with these signaling cascades, autophagy, the already proved inflammatory suppressor, might jointly contribute to $\alpha 7 \mathrm{nAChR}$-mediated anti-neuroinflammation. Further studies need to be done for exploring their potential interaction.

\section{CONCLUSION}

Taken together, we demonstrated the $\alpha 7 \mathrm{nAChR}$-mediated protective roles against neuroinflammation via AMPK-mTORp70S6K related autophagy in monocyte/microglia (Figure S2 in Supplementary Material). We first reported that activating $\alpha 7 \mathrm{nAChR}$ contributed to the alleviation of EAE in severity and inflammatory infiltration. Furthermore, activating $\alpha 7 \mathrm{nAChR}$ significantly led to the induction of autophagy both in spinal cord and spleen from EAE mice and LPS-challenged BV2 microglia. In addition, blockade of autophagy greatly attenuated the $\alpha 7 \mathrm{nAChR}-$ mediated anti-inflammatory effects both in EAE mice and BV2 microglia stimulated with LPS. Finally, we reported that this process involved the AMPK-mTOR-p70S6K signaling pathway. These results demonstrated a novel mechanism for $\alpha 7 \mathrm{nAChR}$ in MS or EAE, which might provide a potential therapeutic target in the treatment of MS and even other inflammation- or autoimmune-related disorders.

\section{ETHICS STATEMENT}

This study was carried out in accordance with the recommendations of the guidelines of the Animal Care Committee of the Second Military Medical University, Shanghai, China. The protocol was approved by the Animal Care Committee of the Second Military Medical University.

\section{REFERENCES}

1. Brandenburg S, Turkowski K, Mueller A, Radev YT, Seidlitz S, Vajkoczy P. Myeloid cells expressinghighlevel ofCD45 are associated with a distinctactivated phenotype in glioma. Immunol Res (2017). doi:10.1007/s12026-017-8915-1

2. Cai Q, Li Y, Pei G. Polysaccharides from Ganoderma lucidum attenuate microglia-mediated neuroinflammation and modulate microglial phagocytosis and behavioural response. J Neuroinflammation (2017) 14:63. doi:10.1186/ s12974-017-0839-0

3. Balez R, Steiner N, Engel M, Munoz SS, Lum JS, Wu Y, et al. Neuroprotective effects of apigenin against inflammation, neuronal excitability and apoptosis in an induced pluripotent stem cell model of Alzheimer's disease. Sci Rep (2016) 6:31450. doi:10.1038/srep31450

4. Hu YY, Wang Y, Liang S, Yu XL, Zhang L, Feng LY, et al. Senkyunolide I attenuates oxygen-glucose deprivation/reoxygenation-induced inflammation in

\section{AUTHOR CONTRIBUTIONS}

$\mathrm{B}-\mathrm{ZS}$ and $\mathrm{PK}$ conducted all experiments in animals and analyzed the data; Z-QX, B-ZH, and M-HC conducted experiments in cells; $\mathrm{CL}$ and $\mathrm{B}-\mathrm{ZS}$ designed the study and wrote the manuscript; $\mathrm{WW}, \mathrm{X}-\mathrm{WC}$, and D-FS revised the manuscript.

\section{ACKNOWLEDGMENTS}

This work was supported by grants from the National Natural Science Foundation of China (81670260) and the State Key Program of National Natural Science of China (81230083).

\section{SUPPLEMENTARY MATERIAL}

The Supplementary Material for this article can be found online at http://journal.frontiersin.org/article/10.3389/fimmu.2017.00553/ full\#supplementary-material.

FIGURE S1 | Blockade of autophagy by bafilomycin A1 attenuates the anti-inflammatory effect of PNU282987 in BV2 microglia stimulated with lipopolysaccharide (LPS). BV2 microglia were treated with bafilomycin A1 (5 nM) for 10 min before the stimulation of PNU282987 (10 $\mu \mathrm{M})$ and LPS (100 ng/ml) for $12 \mathrm{~h}$. (A) Production of IL-6, IL-1 $\beta$, IL-18, and tumor necrosis factor- $\alpha$ (TNF- $\alpha)$ in mRNA level were detected by real-time PCR. The blockade of autophagy process with bafilomycin A1 significantly increased the production of IL-6, IL-1 $\beta, I L-18$, and TNF- $\alpha$ in mRNA level under the stimulation of LPS ( $n=6$ per group). (B) Expressions of LC3-I and LC3-II in protein were detected by Western blotting. Bafilomycin A1 significantly inhibited the effect of PNU282987 on LC3-II/I ratio in LPS-stimulated BV2 microglia ( $n=6$ per group). (C) Quantitative analysis of relative level of LC3-II/I ratio. (D) Cell viability was assessed by CCK-8 assays. The administration of LPS, PNU282987, or bafilomycin A1 did not produce significant effect on cell viability ( $n=6$ per group). ${ }^{\star \star} P<0.01$ vs normal, ${ }^{\#} P<0.01$ vs Veh, ${ }^{8} P P<0.01$ vs control. Veh, vehicle; PNU, PNU282987; bafilomycin A1, Baf A1.

FIGURE S2 | Schematic illustration of the protective mechanism of $\alpha 7 n A C h R$ activation through the induction of autophagy in monocyte/ microglia. (A) Under the occurrence of experimental autoimmune encephalomyelitis (EAE) or the stimulation of lipopolysaccharide (LPS), inflammatory response is greatly triggered in monocyte/microglia which significantly overwhelms the induced protective autophagy, thus leading to the deterioration of EAE severity. (B) However, activating $\alpha 7 n$ AChR by PNU282987, a specific $\alpha 7 \mathrm{nAChR}$ agonist, largely increased the level of autophagy in monocyte/microglia, which contributes to the inhibition of inflammation and consequently the alleviation of EAE, restoring the balance between the protective autophagy and inflammation. PNU, PNU282987.

microglial cells. Brain Res (2016) 1649(Pt A):123-31. doi:10.1016/j.brainres. 2016.08.012

5. Zhou Y, Wu Z, Cao X, Ding L, Wen Z, Bian JS. HNO suppresses LPS-induced inflammation in BV-2 microglial cells via inhibition of NF-kappaB and p38 MAPK pathways. Pharmacol Res (2016) 111:885-95. doi:10.1016/j. phrs.2016.08.007

6. Shao BZ, Wei W, Ke P, Xu ZQ, Zhou JX, Liu C. Activating cannabinoid receptor 2 alleviates pathogenesis of experimental autoimmune encephalomyelitis via activation of autophagy and inhibiting NLRP3 inflammasome. CNS Neurosci Ther (2014) 20:1021-8. doi:10.1111/cns.12349

7. Eixarch H, Calvo-Barreiro L, Montalban X, Espejo C. Bone morphogenetic proteins in multiple sclerosis: role in neuroinflammation. Brain Behav Immun (2017). doi:10.1016/j.bbi.2017.02.019

8. Greenhalgh AD, Passos Dos Santos R, Zarruk JG, Salmon CK, Kroner A, David S. Arginase-1 is expressed exclusively by infiltrating myeloid cells in 
CNS injury and disease. Brain Behav Immun (2016) 56:61-7. doi:10.1016/j. bbi.2016.04.013

9. Ta HM, Le TM, Ishii H, Takarada-Iemata M, Hattori T, Hashida K, et al. Atf6alpha deficiency suppresses microglial activation and ameliorates pathology of experimental autoimmune encephalomyelitis. J Neurochem (2016) 139(6):1124-37. doi:10.1111/jnc.13714

10. Albuquerque EX, Pereira EF, Alkondon M, Rogers SW. Mammalian nicotinic acetylcholine receptors: from structure to function. Physiol Rev (2009) 89:73-120. doi:10.1152/physrev.00015.2008

11. Cheng Q, Yakel JL. The effect of alpha7 nicotinic receptor activation on glutamatergic transmission in the hippocampus. Biochem Pharmacol (2015) 97:439-44. doi:10.1016/j.bcp.2015.07.015

12. Liu C, Su D. Nicotinic acetylcholine receptor alpha7 subunit: a novel therapeutic target for cardiovascular diseases. Front Med (2012) 6:35-40. doi:10.1007/ s11684-012-0171-0

13. Yu JG, Song SW, Shu H, Fan SJ, Liu AJ, Liu C, et al. Baroreflex deficiency hampers angiogenesis after myocardial infarction via acetylcholine-alpha7nicotinic ACh receptor in rats. Eur Heart J (2013) 34:2412-20. doi:10.1093/ eurheartj/ehr299

14. Nizri E, Irony-Tur-Sinai M, Faranesh N, Lavon I, Lavi E, Weinstock M, et al. Suppression of neuroinflammation and immunomodulation by the acetylcholinesterase inhibitor rivastigmine. J Neuroimmunol (2008) 203:12-22. doi:10.1016/j.jneuroim.2008.06.018

15. Nizri E, Irony-Tur-Sinai M, Lory O, Orr-Urtreger A, Lavi E, Brenner T. Activation of the cholinergic anti-inflammatory system by nicotine attenuates neuroinflammation via suppression of Th1 and Th17 responses. JImmunol (2009) 183:6681-8. doi:10.4049/jimmunol.0902212

16. Hao J, Simard AR, Turner GH, Wu J, Whiteaker P, Lukas RJ, et al. Attenuation of CNS inflammatory responses by nicotine involves alpha7 and non-alpha7 nicotinic receptors. Exp Neurol (2011) 227:110-9. doi:10.1016/j. expneurol.2010.09.020

17. Sheng R, Qin ZH. The divergent roles of autophagy in ischemia and preconditioning. Acta Pharmacol Sin (2015) 36:411-20. doi:10.1038/aps.2014.151

18. Ke P, Shao BZ, Xu ZQ, Chen XW, Liu C. Intestinal autophagy and its pharmacological control in inflammatory bowel disease. Front Immunol (2017) 7:695. doi:10.3389/fimmu.2016.00695

19. Shao BZ, Han BZ, Zeng YX, Su DF, Liu C. The roles of macrophage autophagy in atherosclerosis. Acta Pharmacol Sin (2016) 37:150-6. doi:10.1038/ aps.2015.87

20. Wang P, Xu TY, Wei K, Guan YF, Wang X, Xu H, et al. ARRB1/beta-arrestin-1 mediates neuroprotection through coordination of BECN1-dependent autophagy in cerebral ischemia. Autophagy (2014) 10:1535-48. doi:10.4161/ auto. 29203

21. Liang P, Le W. Role of autophagy in the pathogenesis of multiple sclerosis. Neurosci Bull (2015) 31:435-44. doi:10.1007/s12264-015-1545-5

22. Fu L, Huang L, Cao C, Yin Q, Liu J. Inhibition of AMP-activated protein kinase alleviates focal cerebral ischemia injury in mice: interference with mTOR and autophagy. Brain Res (2016) 1650:103-11. doi:10.1016/j.brainres.2016.08.035

23. Park G, Tan JQ, Garcia G, Kang YY, Salvesen G, Zhang ZH. Regulation of histone acetylation by autophagy in Parkinson disease. J Biol Chem (2016) 291:3531-40. doi:10.1074/jbc.M115.675488

24. Wang P, Miao CY. Autophagy in the disorders of central nervous system: vital and/or fatal? CNS Neurosci Ther (2012) 18:955-6. doi:10.1111/cns.12024

25. Lopez A, Lee SE, Wojta K, Ramos EM, Klein E, Chen J, et al. A152T tau allele causes neurodegeneration that can be ameliorated in a zebrafish model by autophagy induction. Brain (2017) 140(4):1128-46. doi:10.1093/brain/ awx005

26. Abdel Fattah E, Bhattacharya A, Herron A, Safdar Z, Eissa NT. Critical role for IL-18 in spontaneous lung inflammation caused by autophagy deficiency. J Immunol (2015) 194:5407-16. doi:10.4049/jimmunol.1402277

27. Wang Q, Zeng P, Liu Y, Wen G, Fu X, Sun X. Inhibition of autophagy ameliorates atherogenic inflammation by augmenting apigenin-induced macrophage apoptosis. Int Immunopharmacol (2015) 27:24-31. doi:10.1016/j. intimp.2015.04.018

28. Zhen C, Feng X, Li Z, Wang Y, Li B, Li L, et al. Suppression of murine experimental autoimmune encephalomyelitis development by 1,25 -dihydroxyvitamin D3 with autophagy modulation.J Neuroimmunol (2015)280:1-7.doi:10.1016/j. jneuroim.2015.01.012
29. Feng X, Hou H, Zou Y, Guo L. Defective autophagy is associated with neuronal injury in a mouse model of multiple sclerosis. Bosn J Basic Med Sci (2017). doi:10.17305/bjbms.2017.1696

30. Jiang Y, Kou J, Han X, Li X, Zhong Z, Liu Z, et al. ROS-dependent activation of autophagy through the PI3K/Akt/mTOR pathway is induced by hydroxysafflor yellow A-sonodynamic therapy in THP-1 macrophages. Oxid Med Cell Longev (2017) 2017:8519169. doi:10.1155/2017/8519169

31. Ye J, Jiang Z, Chen X, Liu M, Li J, Liu N. The role of autophagy in pro-inflammatory responses of microglia activation via mitochondrial reactive oxygen species in vitro. J Neurochem (2017). doi:10.1111/jnc.14042

32. Hou SW, Liu CY, Li YH, Yu JZ, Feng L, Liu YT, et al. Fasudil ameliorates disease progression in experimental autoimmune encephalomyelitis, acting possibly through antiinflammatory effect. CNS Neurosci Ther (2012) 18:909-17. doi:10.1111/cns. 12002

33. Cavone L, Peruzzi B, Caporale R, Chiarugi A. Long-term suppression of EAE relapses by pharmacological impairment of epitope spreading. Br J Pharmacol (2014) 171:1501-9. doi:10.1111/bph.12525

34. Choi AM, Ryter SW, Levine B. Autophagy in human health and disease. NEngl J Med (2013) 368:651-62. doi:10.1056/NEJMra1205406

35. Jiang LB, Lee S, Wang Y, Xu QT, Meng DH, Zhang J. Adipose-derived stem cells induce autophagic activation and inhibit catabolic response to proinflammatory cytokines in rat chondrocytes. Osteoarthritis Cartilage (2016) 24:1071-81. doi:10.1016/j.joca.2015.12.021

36. Chen YL, Song JJ, Chen XC, Xu W, Zhi Q, Liu YP, et al. Mechanisms of pyruvate kinase $\mathrm{M} 2$ isoform inhibits cell motility in hepatocellular carcinoma cells. World J Gastroenterol (2015) 21:9093-102. doi:10.3748/wjg.v21.i30. 9093

37. Shen SY, Zhang Y, Wang Z, Liu R, Gong XG. Bufalin induces the interplay between apoptosis and autophagy in glioma cells through endoplasmic reticulum stress. Int J Biol Sci (2014) 10:212-24. doi:10.7150/ijbs.8056

38. Agarwal S, Tiwari SK, Seth B, Yadav A, Singh A, Mudawal A, et al. Activation of autophagic flux against xenoestrogen bisphenol-A-induced hippocampal neurodegeneration via AMP kinase (AMPK)/mammalian target of rapamycin (mTOR) pathways. J Biol Chem (2015) 290:21163-84. doi:10.1074/jbc. M115.648998

39. Martinet W, De Meyer GR. Autophagy in atherosclerosis. Curr Atheroscler Rep (2008) 10:216-23. doi:10.1007/s11883-008-0034-y

40. Paludan C, Schmid D, Landthaler M, Vockerodt M, Kube D, Tuschl T, et al. Endogenous MHC class II processing of a viral nuclear antigen after autophagy. Science (2005) 307:593-6. doi:10.1126/science.1104904

41. Choi BY, Jang BG, Kim JH, Seo JN, Wu G, Sohn M, et al. Copper/zinc chelation by clioquinol reduces spinal cord white matter damage and behavioral deficits in a murine MOG-induced multiple sclerosis model. Neurobiol Dis (2013) 54:382-91. doi:10.1016/j.nbd.2013.01.012

42. Dello Russo C, Lisi L, Feinstein DL, Navarra P. mTOR kinase, a key player in the regulation of glial functions: relevance for the therapy of multiple sclerosis. Glia (2013) 61:301-11. doi:10.1002/glia.22433

43. Francois A, Terro F, Quellard N, Fernandez B, Chassaing D, Janet T, et al. Impairment of autophagy in the central nervous system during lipopolysaccharide-induced inflammatory stress in mice. Mol Brain (2014) 7:56. doi:10.1186/s13041-014-0056-Z

44. Ke P, Shao BZ, Xu ZQ, Wei W, Han BZ, Chen XW, et al. Activation of cannabinoid receptor 2 ameliorates DSS-induced colitis through inhibiting NLRP3 inflammasome in macrophages. PLoS One (2016) 11:e0155076. doi:10.1371/ journal.pone.0155076

45. Jeong JK, Park SY. Melatonin regulates the autophagic flux via activation of alpha-7 nicotinic acetylcholine receptors. J Pineal Res (2015) 59:24-37. doi:10.1111/jpi.12235

46. Jeong JK, Park SY. Neuroprotective effect of cellular prion protein (PrPC) is related with activation of alpha7 nicotinic acetylcholine receptor (alpha7nAchR)mediated autophagy flux. Oncotarget (2015) 6:24660-74. doi:10.18632/ oncotarget. 4953

47. Fan T, Huang Z, Chen L, Wang W, Zhang B, Xu Y, et al. Associations between autophagy, the ubiquitin-proteasome system and endoplasmic reticulum stress in hypoxia-deoxygenation or ischemia-reperfusion. Eur J Pharmacol (2016) 791:157-67. doi:10.1016/j.ejphar.2016.08.026

48. Klionsky DJ, Abdelmohsen K, Abe A, Abedin MJ, Abeliovich H, Acevedo Arozena A, et al. Guidelines for the use and interpretation of assays for 
monitoring autophagy (3rd edition). Autophagy (2016) 12:1-222. doi:10.1080/ 15548627.2015.1100356

49. Takanezawa Y, Nakamura R, Sone Y, Uraguchi S, Kiyono M. Atg5-dependent autophagy plays a protective role against methylmercury-induced cytotoxicity. Toxicol Lett (2016) 262:135-41. doi:10.1016/j.toxlet.2016.09.007

50. Kawamata J, Shimohama S. Stimulating nicotinic receptors trigger multiple pathways attenuating cytotoxicity in models of Alzheimer's and Parkinson's diseases. J Alzheimers Dis (2011) 24(Suppl 2):95-109. doi:10.3233/JAD-2011-110173

51. Lykhmus O, Mishra N, Koval L, Kalashnyk O, Gergalova G, Uspenska K, et al. Molecular mechanisms regulating LPS-induced inflammation in the brain. Front Mol Neurosci (2016) 9:19. doi:10.3389/fnmol.2016.00019

52. Wang YY, Hu Ch F, Li J, You X, Gao FG. Increased translocation of antigens to endosomes and TLR4 mediated endosomal recruitment of TAP contribute to nicotine augmented cross-presentation. Oncotarget (2016) 7:38451-66. doi:10.18632/oncotarget.9498

53. Xu ZQ, Shao BZ, Ke P, Liu JG, Liu GK, Chen XW, et al. Combined administration of anisodamine and neostigmine rescued acute lethal crush syndrome through alpha7nAChR-dependent JAK2-STAT3 signaling. Sci Rep (2016) 6:37709. doi:10.1038/srep37709

54. Zhang Q, Lu Y, Bian H, Guo L, Zhu H. Activation of the alpha7 nicotinic receptor promotes lipopolysaccharide-induced conversion of M1 microglia to M2. Am J Transl Res (2017) 9:971-85.

Conflict of Interest Statement: The authors declare that the research was conducted in the absence of any commercial or financial relationships that could be construed as a potential conflict of interest.

Copyright () 2017 Shao, Ke, Xu, Wei, Cheng, Han, Chen, Su and Liu. This is an open-access article distributed under the terms of the Creative Commons Attribution License (CC BY). The use, distribution or reproduction in other forums is permitted, provided the original author(s) or licensor are credited and that the original publication in this journal is cited, in accordance with accepted academic practice. No use, distribution or reproduction is permitted which does not comply with these terms. 